\title{
Prediction of recurrent geomagnetic disturbances by using adaptive filtering
}

\author{
X.-Y. Zhou ${ }^{1}$ and F.-S. Wei ${ }^{2}$ \\ ${ }^{1}$ Institute of Geophysics, Chinese Academy of Sciences, Beijing 100101, P.R. China \\ ${ }^{2}$ Center for Space Science and Applied Research, Chinese Academy of Sciences, Beijing 100080, P.R. China
}

(Received December 15, 1997; Revised July 28, 1998; Accepted July 28, 1998)

\begin{abstract}
Recurrent geomagnetic disturbances are an important part of geomagnetic activities, which are associated with the neutral sheet structure in the heliosphere and the activities of long lived solar coronal holes. Another significant character is the periodic activities recorded by geomagnetic indices. In this paper an algorithm-Adaptive Filtering (AF), is introduced to forecast recurrent geomagnetic events based on the geomagnetic $K$ index. Adaptive filtering can deal with nonstationary data and can adapt to changes in the data pattern. Therefore it is a very helpful method for forecasting the geomagnetic disturbances and the disturbances in the interplanetary space. By using AF technique a prediction for whole Bartels rotation can be obtained when output length is taken as 27-point. For recurrent periods the prediction efficiency is about $30 \%$, the correlation coefficient is 0.55 . For nonrecurrent periods the prediction efficiency and correlation coefficient decrease obviously, but the standard variance does not change very much.
\end{abstract}

\section{Introduction}

The prediction of geomagnetic disturbance is a presentday matter of significant importance to the many scientific investigations, military affairs and commercial concerns. Great efforts have been made by many scientific institutes and services in this area for long time (Joselyn, 1986; Marubashi, 1989). Some prediction techniques including numerical simulation (Akasofu and Fry, 1986), filtering (Iyemori et al., 1979; Clauer et al., 1983; McPherron et al., 1984) and artificial intelligence (Lundstedt, 1989, 1992; Freeman et al., 1993; Wu and Lundstedt, 1996) have been developed or are developing. These methods are mainly based on the fact that the magnetosphere would respond to the disturbed solar wind environment or solar activity. From the point of view of prediction, the geomagnetic disturbances can be divided into two catalogues: transient event and recurrent event. The transient geomagnetic disturbance results from the transient solar activity; this kind of solar activity can result in the solar wind disturbance, such as magnetic cloud, interplanetary shock and discontinuity (Gosling et al., 1975; Schwenn, 1986; Wilson and Hildner, 1986; Tang et al., 1989 and Eselevich, 1990) which can lead to the transient geomagnetic disturbance (Chao and Lepping, 1974; Smith et al., 1986; Wilson, 1987; Gosling et al., 1990; Tsurutani et al., 1990 and Jacobsen et al., 1991). This can be seen as a spatial variation of a disturbance initiated at the Sun then propagating to the Earth through interplanetary space. At different positions this disturbance appears with different properties and characters because of its interaction with the local environment. A typical and well identified solar-terrestrial transient event is the recent Jan 6-11, 1997 event.

On the other hand the recurrent geomagnetic disturbance is associated with the long-lived solar coronal hole and the he-

Copy right (C) The Society of Geomagnetism and Earth, Planetary and Space Sciences (SGEPSS); The Seismological Society of Japan; The Volcanological Society of Japan; The Geodetic Society of Japan; The Japanese Society for Planetary Sciences. liospheric current sheet structure (Neupert and Pizzo, 1974; Hansen et al., 1976; Sheeley et al., 1976; Burlaga and Lepping, 1977). And recently it is refined by emphasizing the importance of stream-stream interaction and coronal mass ejection (Crooker and Cliver, 1994). This recurrence is an evidence for the fact that geomagnetic activity reflects the solar rotation period of approximately 27 days (Bartels, $1932,1934)$, or the existence of a two stream structure in the solar wind with 13.5 day periodicity (Bame et al., 1976; Fenimore et al., 1978; Mursula and Zieger, 1996). No mater how long the recurrent period, the recurrence is a temporal character of geomagnetic activity. It can be seen as a historical variation of geomagnetic indices, such as in $C 9$ and $K$ etc. A very significant recurrent period is from December 1993 to June 1994, which maintained about eight Bartels rotations. Sargent (1986) introduced a 27-day recurrent index by comparing $a a$ index of two successive Bartels rotations. He found that although the recurrence does not always exist, there is apparently a long recurrent period during each sunspot cycle especially at the decline and minimum phase. Therefore during this interval the recurrence provides the possibilities for analyzing and predicting this kind of geomagnetic disturbance.

The prediction of the transient geomagnetic disturbance is mainly based on how the magnetosphere responds to the solar wind disturbance and how the solar wind couples with magnetosphere. The inputs and outputs of prediction models are different parameters describing the different disturbed characters at corresponding spatial position. For example, in the prediction of a magnetic storm by using the Elman networks (Wu and Lundstedt, 1996), solar wind parameters are used as inputs and Dst index is the output. For the recurrent geomagnetic disturbance, the prediction is focused on when the recurrence can work as a forecasting tool, how to identify the recurrent character and how to extract the period of the recurrent activity. At this point, the input and output 
are the same parameter, but corresponding to different time. Generally the historical record before time $t$ for a parameter is used as input. The output is the value of the same parameter at $t+i, i=1,2, \ldots$.

In this paper Adaptive Filtering (AF) is used for forecasting the recurrent geomagnetic activities. The major advantages of a AF technique over other methods of time series analysis are its conceptual and computational simplicity. The objective of a filter, like most other time series methods, is to separate the noise from the signal or pattern in order to discover the true generating process. The equations used by a AF in accomplishing this objective can be simply stated, updated recursively, and are of infinite memory. The generalized AF with its many attractive features and its favorable performance, deserves consideration as a forecasting technique for a wide range of time series cases. A disadvantages of AF during the iterative procedure is that it can be computationally time consuming, even though the basic sequence is straight forward. But it can be corrected by the use of the Yule-Walker equations (Box and Jenkins, 1976).

In this work, we focus on mid-term (one Bartels rotation ahead) prediction of $K p$ and Beijing local $K$ index. The $K$ index obtained from Beijing geomagnetic observatory at Ming Tomb, here is abbreviated as $K_{\mathrm{B}}$ index. It is one of the most common geomagnetic indices used in monitoring and forecasting the geomagnetic activity in China. $K_{\mathrm{BS}}$ is a summation of eight 3-hour $K_{\mathrm{B}}$ to describe daily level of geomagnetic activities in Beijing area.

\section{Adaptive Filtering Method}

One of common time-series analysis methods-adaptive filtering is used for the practical prediction of $K p$ and $K_{\mathrm{BS}}$ indices. The objective of an adaptive filter is, going with filtering, to estimate and rectify the model parameters (online) continuously by means of the new observations, so that predictions closely match the observed data. Therefore, the error of state estimation can be decreased and the filtering precision can be improved. Besides the major advantage of conceptual and computational simplicity of this technique, it also can separate the noise in the time series from the real signal or pattern in order to discover the implied process guiding the forecasting.

As methodology for time series forecasting, adaptive filtering bases its forecast on a weighted sum of past observations, viz.,

$$
\begin{aligned}
& \hat{X}_{t}=\phi_{1 t} X_{t-1}+\phi_{2 t} X_{t-2}+\cdots+\phi_{p t} X_{t-p}+e_{t}, \\
& t=p+1, p+2, \ldots, n
\end{aligned}
$$

This equation represents an autoregressive (AR) model to process. One major difference between adaptive filtering and the AR models is that the parameters, $\phi_{1 t}, \phi_{2 t}, \ldots$, of the latter models are fixed, while those of adaptive filtering are not. This enables adaptive filtering to deal with nonstationary data and to adapt to changes in the data pattern (by updating the model parameters as new data become available) much better than is possible for fixed parameter models.

Modification of the adaptive filtering parameters is done with the following equation

$$
\phi_{i t}^{\prime}=\phi_{i t}+2 k e_{t}^{*} X_{t-i}^{*}
$$

where $i=1,2, \ldots, p, e_{t}^{*}$ and $X_{t-i}^{*}$ are the standardized values used in Eq. (1), $k$ is a learning constant (Makridakis and Wheelwright, 1977).

This expression provides an easily understood and computationally simple updating formula for the parameters of Eq. (1). It also has the advantage of including a learning constant, $k$, which determines the speed by which the parameters of the model are adapted. Expression (2) can be interpreted in a Bayesian sense, where $\phi_{i t}$, the old information about the AR process (1), is combined with new information (the residual or error of the most recent forecast) to form the updated $\phi_{i t}^{\prime}$.

The relationship stated in Eq. (2) can then be used to obtain an improved set of weights as more information becomes available. This is done by using Eq. (1) with the first $p$ data points to calculate $\hat{X}_{t}$ and $e_{t}$. These values and the appropriate $X_{i}$ can be substituted in to Eq. (2) to determine an improved set of weights, $\Phi^{\prime}$. This sequence of steps can then be repeated by dropping the first data point in the set of $p X$ values and adding the next data point, $(p+1)$.

The subjective feelings of the forecaster can be incorporated in the value of $k$. For example, when the user expects a continuation of the basic data pattern, a "normal" value of $k$, such as $1 / p$, can be used. If the forecaster expects a change in the data pattern, the value of $k$ might be increased. Whereas a change in the amount of randomness, but no basic change in the pattern, might lead the forecaster to decrease the value of $k$. This is executed by a parameter of weight $L$ in the code. When $L$ is bigger, such as $L=60$, less change in the data pattern and an increased change in the amount of randomness. As $L$ is decreased the opposite situation happened. In our prediction experiment different $L$ has been used to check the output variations. But for routine prediction and all the results shown in this paper, $L$ is 30 .

We use $R, S t$ and $P E$ to describe the precision of a prediction. They are defined as follows. The correlation coefficient

$$
R=\frac{\sum_{i=1}^{n}\left[\left(X_{i}-\hat{X}_{i}\right)\left(\hat{X}_{i}-\overline{\hat{X}}_{i}\right)\right]}{\sqrt{\sum_{i=1}^{n}\left(X_{i}-\bar{X}_{i}\right)^{2} \sum_{i=1}^{n}\left(\hat{X}_{i}-\overline{\hat{X}}_{i}\right)^{2}}}
$$

then the standard variation

$$
S t=\left(\frac{1}{n} \sum_{i=1}^{n}\left(X_{i}-\hat{X}_{i}\right)^{2}\right)^{1 / 2} .
$$

The prediction efficiency $P E=1-A R V, A R V$ is the average relative variance and defined as (Clauer et al., 1983):

$$
A R V=\frac{\sum_{i=1}^{n}\left(X_{i}-\hat{X}_{i}\right)^{2}}{\sum_{i=1}^{n}\left(X_{i}-\bar{X}_{i}\right)^{2}}
$$

where $X_{i}$ and $\bar{X}_{i}$ are the observations and their average, $\hat{X}_{i}$ and $\overline{\hat{X}}_{i}$ are the predictions and their average.

These are three complemental parameters describing comprehensively the goodness of a prediction result. $R$ is the correlation coefficient between the observed and the predicted 


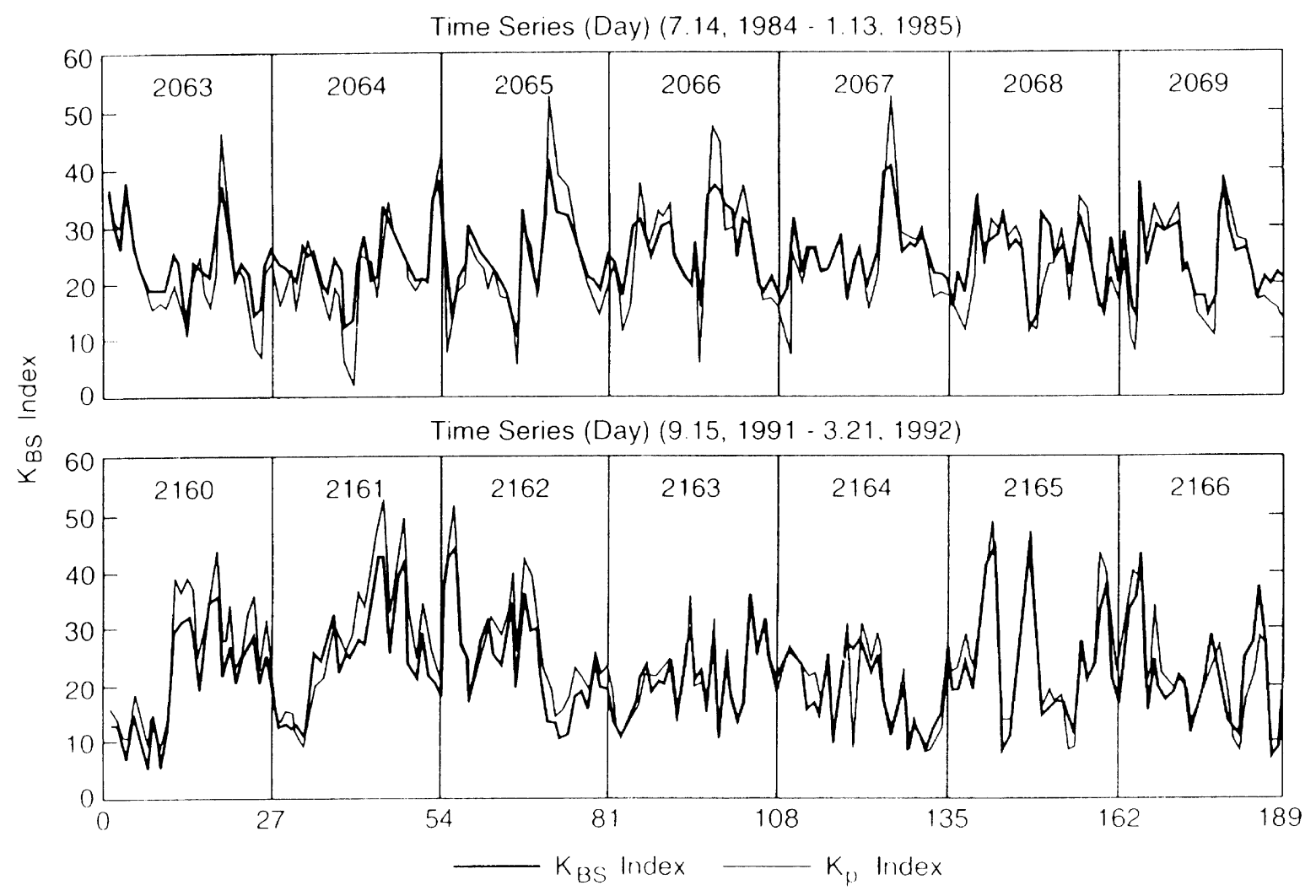

Fig. 1. Comparison between daily summation of $K_{\mathrm{B}}$ and $K p$ index.

values. But even for a complete correlated prediction, when $R=1$, there still might be a discrepancy between the observation and prediction. For example, two curves with the same variance $(R=1)$ can be complete overlapped when there is no distance between them $(S t=0)$. While they are separated when there is a distance between them $(S t>0)$. The quantity of the distance is described by $S t$. The less of $S t$ is, the better of the prediction. $P E$ shows the ability to forecast the variability of the observation. The best prediction should have $P E=100 \%$ or approaches to this value.

\section{Prediction Results and Discussions}

In this work $\sum K p$ and $K_{\mathrm{BS}}$ (summation for daily eight $K p$ or $K_{\mathrm{B}}$ ) are the predicted objects and also are inputs. Figure 1 is the comparison between $\sum K p$ and $K_{\mathrm{BS}}$. The upper panel includes seven Bartels rotations near solar minimum of cycle 21 , the bottom panel is at the peak of solar cycle 22. It is clear that the variation of $K_{\mathrm{BS}}$ is less than $\sum K p$. The difference, perhaps, is due to the inherent weightage for night time in deriving $K p$ and the absence of the same in $K_{\mathrm{B}}$.

Because a summation of each day's 8 digits of $K p$ (or $\left.K_{\mathrm{B}}\right)$ has been made to describe active level of each day, an identification, for example, of 00000008 and 11111111 (with the same summations) can not be given by $\sum K p$ (or $K_{\mathrm{BS}}$ ). But actually most significant disturbances should have accompanied premonitory variations. These premonitory variations provide some possibility for the prediction. The mid-term prediction in this paper is more emphasized on the prediction of geomagnetic varying tendency during the near future.
Generally $\sum K p\left(K_{\mathrm{BS}}\right)$ index can be a good indicator for this tendency as shown in our prediction results. As to the extreme situation of 00000008 , it, at very large extent, is unpredictable.

The time periods used for the prediction experiment in sunspot maximum, sunspot decline and minimum are shown in Table 1. All rotation numbers are of Bartels rotation. The length of input data is six rotations of $\sum K p$ or $K_{\mathrm{BS}}$. The corresponding output prediction is one rotation, 27 days. $R$, $S t$ and $P E$ are calculated as comparing the predictions with observations. The average prediction results of $\sum K p$ and $K_{\mathrm{BS}}$ are given by two histograms in Fig. 2 . For both indices although the average $R$ decreases obviously from recurrent to nonrecurrent periods, $S t$ does not change very much, even $S t$ decreases for $K_{\mathrm{BS}}$. That means for nonrecurrent periods the one rotation ahead prediction of background variation of the geomagnetic activity is almost the same with that of recurrent periods. A typical example for this situation can be seen from Fig. 3. Here $R$ is low, even $P E<0$, but $S t$ is also very low. So the prediction shows very well the background variation for the whole rotation.

According to Fig. 2, it is hard to say which prediction, for $\sum K p$ or $K_{\mathrm{BS}}$, is better because of the different varying of the three parameters. For the prediction of $K_{\mathrm{BS}}$, the low $P E$ and $S t$ is mainly because of the less variability of $K_{\mathrm{BS}}$ as shown in Fig. 1. It can be inferred that the prediction for $A p$ index by use AF technique should have larger $P E$ and $S t$ because of the larger varying amplitude in $A p$ index at the disturbed time. While parameter $R$ is not affected by the 
Table 1. Time periods used in the prediction experiment.

\begin{tabular}{lll}
\hline Index & Sunspot maximum & Sunspot decline and minimum \\
\hline \multirow{3}{*}{$K p$} & No. 2160-2166 & No. 1921-1948 (Jan 14, 1974-Feb 8, 1976) \\
& $($ Sep 15, 1991-Mar 21, 1992) & No. 2058-2077 (Mar 1, 1984-Aug 22, 1985) \\
& & No. 2218-2231 (Dec 29, 1995-Jan 9, 1997) \\
\hline \multirow{2}{*}{$K_{\text {BS }}$} & No. 2160-2166 & No. 2063-2069 (Jul 14, 1984-Jan 18, 1985) \\
& $($ Sep 15, 1991-Mar 21, 1992) & No. 2182-2231 (May 1, 1993-Feb 5, 1997) \\
\hline
\end{tabular}

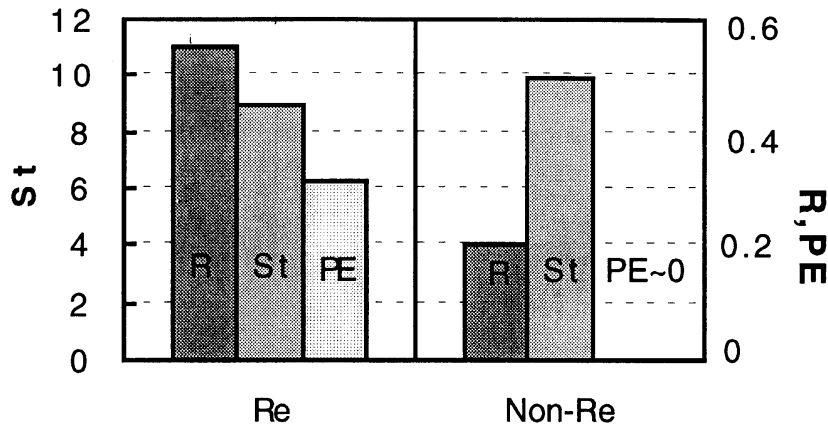

(a)

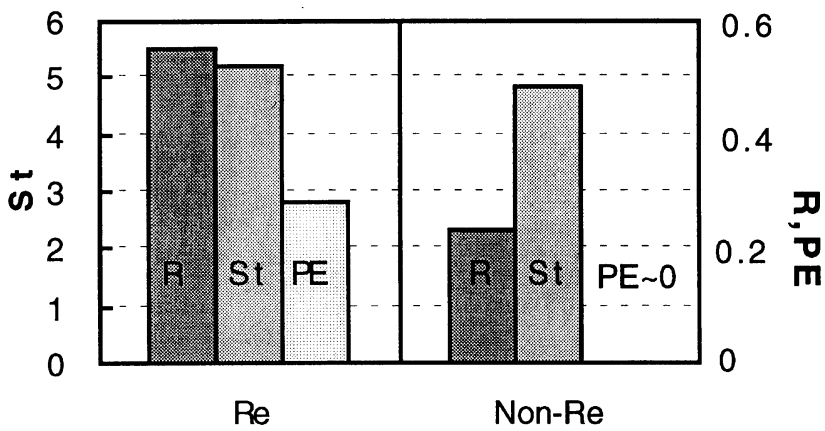

(b)

Fig. 2. (a) An average prediction result of $\sum K p$. (b) An average prediction result of $K_{\mathrm{BS}}$. Re: recurrent periods; Non-Re: nonrecurrent periods.

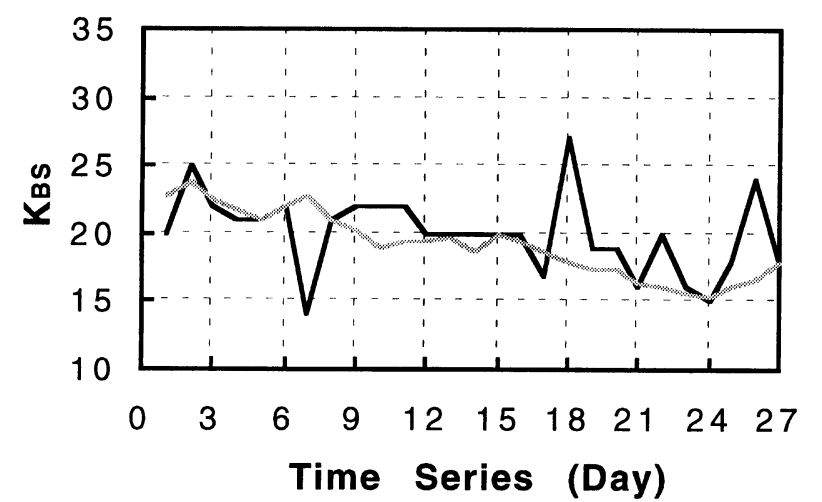

Fig. 3. The comparison between observation and prediction of $K_{\mathrm{BS}}$ (No. 2223: $R=0.3359, S t=3.16, P E<0$ ). The darker line is for observation and the lighter line is for prediction. (It is the same in Figs. 4 and 5).

varying amplitude very much.

The best and worst prediction results for $K_{\mathrm{BS}}$ and $\sum K p$ during the periods of recurrence and nonrecurrence are given in Figs. 4 and 5 respectively. $R, S t$ and $P E$ are shown in figure captions. In this paper, recurrent period means that the correlation coefficient of $K$ index between the two succeeding rotations is higher than 0.5 and it can exist at least two or more rotations continuously. From these figures we can know the precision of this quantitative prediction more clearly and more perceptually. For real-time prediction all the predictions are made at the first day of a Bartels rotation, and then $R, S t$ and $P E$ of this rotation can be calculated at the first day of next Bartels rotation.

As shown by expressions (3)-(5), $R, S t$ and $P E$ describe the goodness of the prediction from different aspects. $P E$ describes the ability to predict the variation of the observation and is affected by the varied amplitude of the observations. For an extreme example, if the prediction is just the average of the observation, no matter how big of the varied amplitude, the prediction efficiency should be zero. However for very small varied amplitude of the observation this prediction should be a good prediction with very low standard variance, even though the prediction efficiency is zero. So it can not say they are bad prediction in rotation No. 2222 and No. 2223 because of the small or negative PE. Also comparing Figs. 4(a) and 5(a), the prediction in Fig. 4(a) should be better with higher $R$ and lower $S t$, but its $P E$ is lower than that of Fig. 5(a). This is because the variance of $K_{\mathrm{BS}}$ in No. 2199 is smaller than that of $K p$ in No. 1932.

The length of input data used in our prediction is 6 rotations. Calculated result and experience have shown that the updated data length can be chosen as 4 to 7 rotations. Too short or too long input data will make the recurrent information not clear. We use one $K_{\mathrm{BS}}$ or $\sum K p$ for each day. So the output, the one rotation ahead prediction, is a 27-point data series. Actually, according to the realistic requirement, the prediction length may be chosen as 1 day, 13 days or even one month. If prediction output is selected as 1 day, and then using this 1 day prediction as input for next day predic- 


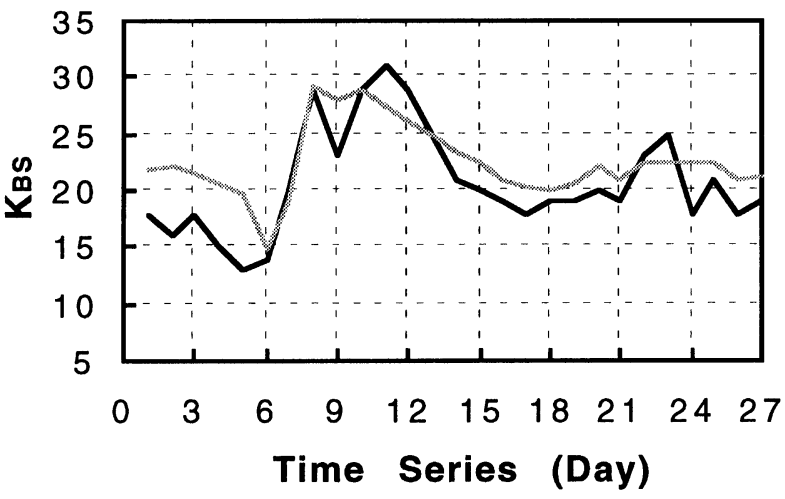

(a)

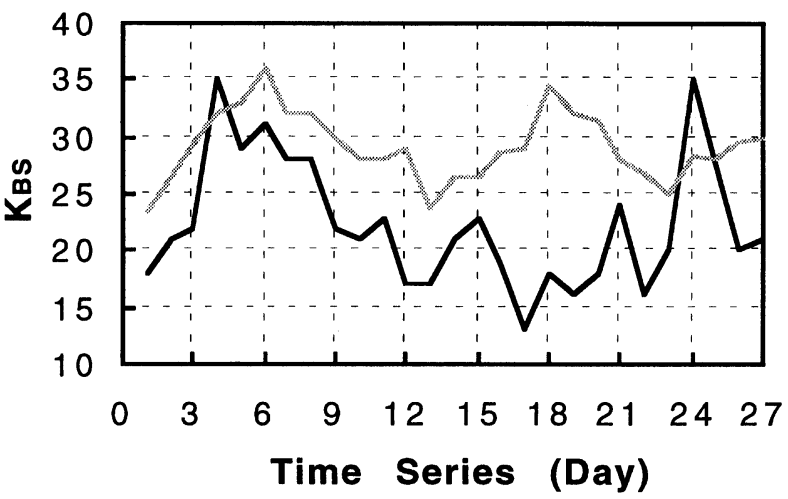

(b)

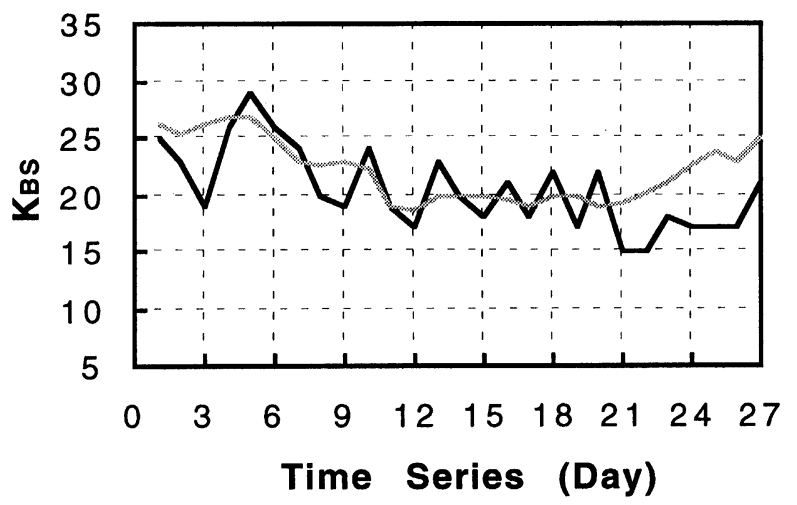

(c)

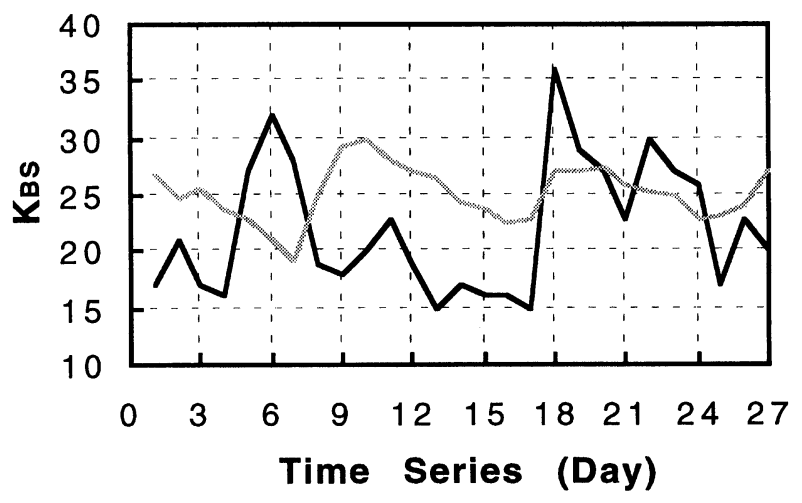

(d)

Fig. 4. (a) The best prediction for recurrent period (No. 2199: $R=0.8242, S t=3.14, P E=54.0 \%$ ). (b) The worst prediction for recurrent period (No. 2210: $R=0.3702, S t=8.56, P E<0)$. (c) The best prediction for nonrecurrent period (No. 2222: $R=0.5319, S t=3.16, P E=8.3 \%$ ). $($ d) $T h e$ worst prediction for nonrecurrent period (No. 2205: $R=-0.0780, S t=7.10, P E<0$ ).

tion etc., eventually one rotation ahead prediction also can be obtained after this iterative procedure. But this should not bring in higher prediction accuracy than one rotation prediction directly given by a 27-point output. Because when you use 1-point prediction output as input the error of this prediction will be maintained or even enlarged. So we suggest that for multi-point prediction, just directly select the output number as long as the prediction length you need.

\section{Conclusions}

Sometimes the geomagnetic activity basically controlled by the solar wind are recurrent. This recurrence can provide sufficient information for forecasting the geomagnetic activities in near future. In this paper an Adaptive Filtering technique is applied for this purpose. For both $\sum K p$ and $K_{\mathrm{BS}}$ during the recurrent periods the prediction efficiency are about $30 \%$, the correlation coefficient is 0.55 .

During the nonrecurrent periods, $R$ and $P E$ are very low. Basically we do not suggest to use this technique to predict the geomagnetic activities without recurrency. But Fig. 2 shows a very interesting character, there is no big change at $S t$ between the recurrent and nonrecurrent periods. That means to a certain extent AF technique can predict the variation tendency of geomagnetism even during the nonrecurrent interval. So if there is no big variations or disturbances during a nonrecurrent period, AF can still be a good method to predict the variation tendency of a whole rotation.

As AF technique is applied to the temporal variation of the geomagnetic disturbances in this work, an improved prediction can definitely be obtained when a prediction is superposed, which is based on the spatial variation. For instance in Fig. 3, an unpredictable peak at 18th day can probably be improved by superposing the prediction based on the spatial variation, in which the controlling elements in the solar wind are used as inputs.

Three parameters are used for describing a prediction result from differenct aspects. They are correlation coefficient $R$ and standard variation $S t$ and prediction efficiency $P E . P E$ brings a relative comprehensive efficiency of the prediction. But none of them is a good single standard for the prediction results.

Prediction of geomagnetic disturbance is all along a very complicate problem on the physics and techniques. Various prediction methods have been, are being or will be developed based on different physical mechanism, algorithm techniques and different indices. How to combine them together is still an open question, although it is a well known future objective. 


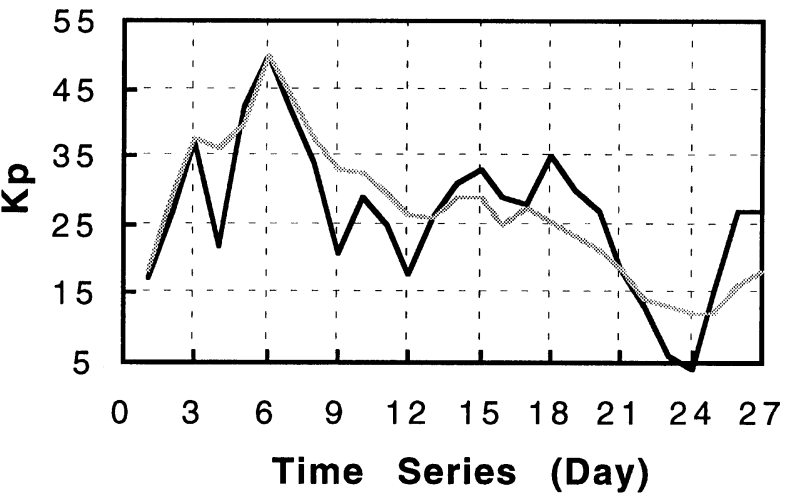

(a)

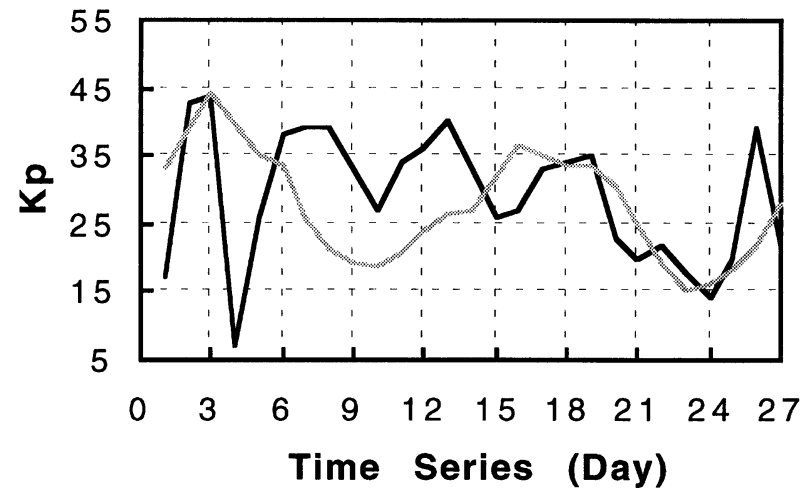

(b)

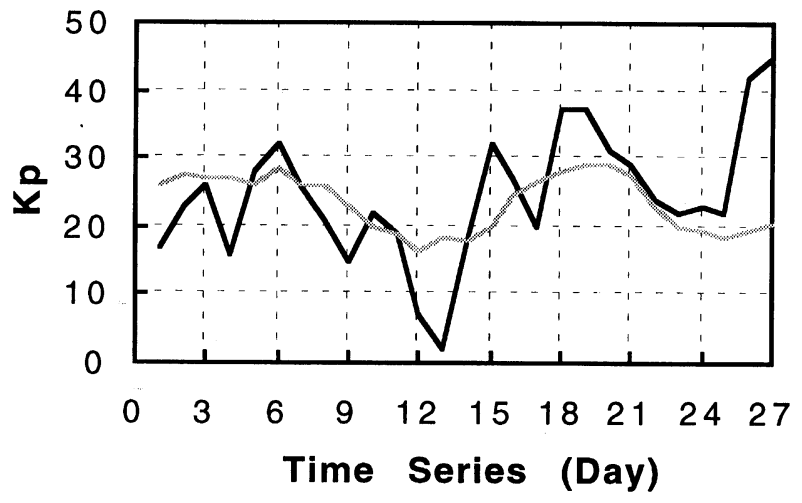

(c)

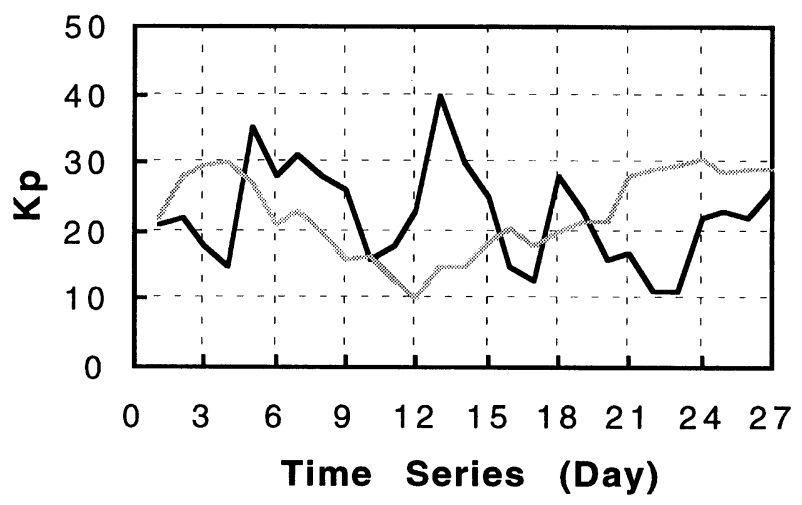

(d)

Fig. 5. (a) The best prediction for recurrent period (No. 1932: $R=0.8005, S t=6.38, P E=61.6 \%$ ). (b) The worst prediction for recurrent period (No. 1930: $R=0.1868, S t=11.24, P E<0)$. (c) The best prediction for nonrecurrent period $($ No. $2064: R=0.3450, S t=9.11, P E=10.6 \%)$. (d) The worst prediction for nonrecurrent period (No. 2076: $R=-0.3245, S t=11.14, P E<0$ ).

Acknowledgments. We would like to thank Prof. K.-K. Tschu for his helpful discussions. We thank two referees for their comments and suggestions on revising the manuscript. This work was finished at the Institute of Geophysics, the Chinese Academy of Sciences and was supported by the National Science Foundation of China under the grant of HT 49384007.

The editor thanks G. K. Rangarajan and another referee for their assistance in evaluating this paper.

\section{References}

Akasofu, S.-I. and C. Fry, A first generation numerical geomagnetic storm prediction scheme, Planet. Space Sci., 34(1), 77-92, 1986.

Bame, S. J., J. R. Asbridge, W. C. Feldman, and J. T. Gosling, Solar cycle evolution of high-speed solar wind streams, Astrophys. J., 207(3), 977980, 1976.

Bartels, J., Terrestrial magnetic activity and its relations to solar phenomena, J. Geophys. Res., 37(1), 1-52, 1932.

Bartels, J., Twenty-seven day recurrencies in terrestrial-magnetic and solar activity, 1923-1933, J. Geophys. Res., 39(1), 201-202, 1934.

Box, G. E. P. and G. M. Jenkins, Time-Series Analysis: Forecasting and Control, Holden-Day, San Francisco, 1976.

Burlaga, L. F. and R. P. Lepping, The causes of recurrent geomagnetic storms, Planet. Space Sci., 25(12), 1151-1160, 1977.

Chao, J. K. and R. P. Lepping, A correlative study of SSC's, Interplanetary shocks, and Solar activity, J. Geophys. Res., 79(3), 1799-1807, 1974.

Clauer, R., R. L. McPherron, and C. Searls, Solar wind control of the low latitude asymmetric magnetic disturbance field, J. Geophys. Res., 88(A4), 2123-2130, 1983.

Crooker, N. U. and E. W. Cliver, Postmodern view of M-regions, J. Geophys.
Res., 99(A12), 23383-23390, 1994.

Eselevich, V. G., Solar flares: Geoeffectiveness and the possibility of a new classification, Planet. Space Sci., 38(2), 189-206, 1990.

Fenimore, E. E., J. R. Asbridge, S. J. Bame, W. C. Feldman, and J. T. Gosling, The power spectrum of the solar wind speed for periods greater than 10 days, J. Geophys. Res., 83(A9), 4353-4357, 1978.

Freeman, J., A. Nagai, P. Reiff, W. Denig, S. Gussenhoven-Shea, M Heinemann, F. Rich, and M. Hairston, The use of neural networks to predict magnetospheric parameters for input to a magnetospheric forecast model, in Proceedings of Artificial Intelligence Applications in SolarTerrestrial Physics Workshop in Lund Sweden, September 22-24, 1993, edited by J. A. Joselyn, H. Lundstedt, and J. Trollinger, pp. 167-174, 1993.

Gosling, J. T., E. Hildner, R. M. MacQueen, R. H. Munro, A. I. Poland, and C. L. Ross, Direct observations of a flare related coronal and solar wind disturbance, Solar Phys., 40(2), 439-448, 1975.

Gosling, J. T., S. J. Bame, D. J. McComas, and J. L. Phillips, Coronal mass ejections (CME) and large geomagnetic storms, Geophys. Res. Lett., 17(7), 901-904, 1990

Hansen, R. T., S. F. Hansen, and C. Sawyer, Long-lived coronal structure and recurrent geomagnetic patterns in 1974, Planet. Space Sci., 24(4), 381-388, 1976.

Iyemori, T., H. Maeda, and T. Kamei, Impulse response of geomagnetic indices to interplanetary magnetic field, J. Geomag. Geoelectr., 31(1), 1-9, 1979.

Jacobsen, B., P. E. Sandholt, B. Lybekk, and A. Egeland, Transient auroral events near midday: Relationship with solar wind/magnetosheath, $J$. Geophys. Res., 96(A2), 1327-1336, 1991.

Joselyn, J. A., Real-time prediction of global geomagnetic activity, in Solar 
Wind-Magnetosphere Coupling, edited by Y. Kamide and J. A. Slavin, pp. 127-138, Terra, Tokyo, Japan, 1986.

Lundstedt, H., An inductive expert system for solar-terrestrial predictions, in Proceeding of Solar-Terrestrial Prediction Workshop, pp. 126-129, Leura, Australia, 1989

Lundstedt, H., Neural network and prediction of solar-terrestrial effects, Planet. Space Sci., 40(4), 457-464, 1992.

Makridakis, S. and S. C. Wheelwright, Adaptive filtering: an integrated autoregressive/moving average filter for series forecasting, Oper. Res. Quarterly, 28(2,ii), 425-437, 1977.

Marubashi, K., The space weather forecast program, Space Sci. Rev., 51(1/2), 197-214, 1989.

McPherron, R. L., R. A. Fay, C. R. Garrity, L. F. Bargatze, D. N. Baker, C. R. Clauer, and C. Searls, Coupling of the solar wind to measures of magnetic activity, in Proc. Conf. Achievement of the IMS, Graz. Austria, ESA SP-217, pp. 161-170, 1984.

Mursula, K. and B. Zieger, The 13.5-day periodicity in the Sun, solar wind, and geomagnetic activity: The last three solar cycles, J. Geophys. Res., 101(A12), 27077-27090, 1996

Neupert, W. M. and V. Pizzo, Solar coronal holes as sources of recurrent geomagnetic disturbances, J. Geophys. Res., 79(25), 3701-3709, 1974.

Sargent, H. H., III, The 27-day recurrence index, in Solar Wind-Magnetosphere Coupling, edited by Y. Kamide and J. A. Slavin, pp. 143-154, Terra, Tokyo, Japan, 1986.

Schwenn, R., Relationship of coronal transients to interplanetary shocks:
3D aspects, Space Sci. Rev., 44(1/2), 139-168, 1986.

Sheeley, N. R., Jr., J. W. Harvey, and W. C. Feldman, Coronal holes, solar wind streams, and recurrent geomagnetic disturbances 1973-1976, Sol. Phys., 49(2), 271-278, 1976.

Smith, E. J., J. A. Slavin, R. D. Zwickl, and S. J. Bame, Shocks and storm sudden commencements, in Solar Wind-Magnetosphere Coupling, edited by Y. Kamide and J. A. Slavin, pp. 345-357, Terra, Tokyo, Japan, 1986. Tang, F., B. T. Tsurutani, W. D. Gonzalez, S.-I. Akasofu, and E. J. Smith, Solar sources of interplanetary southward Bz events responsible for major magnetic storms (1978-1979), J. Geophys. Res., 94(A4), 3535-3541, 1989.

Tsurutani, B. T., B. E. Glodstein, E. J. Smith, W. D. Gonzalez, F. Tang, S.-I. Akasofu, and R. R. Anderson, The interplanetary and solar causes of geomagnetic activity, Planet. Space Sci., 38(1), 109-126, 1990.

Wilson, R. M., Geomagnetic response to magnetic clouds, Planet. Space Sci., 35(3), 329-335, 1987.

Wilson, R. M. and E. Hildner, On the association of magnetic clouds with disappearing filaments, J. Geophys. Res., 91(A5), 5867-5872, 1986.

$\mathrm{Wu}$, J.-G. and H. Lundstedt, Prediction of geomagnetic storms from solar wind data using Elman recurrent neural networks, Geophys. Res. Lett., 23(4), 319-322, 1996.

X.-Y. Zhou (e-mail: zhou@jplsp.jpl.nasa.gov) and F.-S. Wei 